Check for updates

Cite this: RSC Adv., 2017, 7, 29618

Received 19th February 2017

Accepted 28th May 2017

DOI: $10.1039 / c 7 r a 02065 a$

rsc.li/rsc-advances

\section{Non-thermal acoustic treatment as a safe alternative to thermosensitive liposome-involved hyperthermia for cancer therapy}

\author{
Wooram Um, $\dagger^{\mathrm{a}}$ Seunglee Kwon, $\hat{\dagger}^{\mathrm{b}}$ Dong Gil You, ${ }^{\mathrm{b}}$ Jae Min Cha, ${ }^{\mathrm{C}}$ Hyun Ryoung Kim ${ }^{\mathrm{d}}$ \\ and Jae Hyung Park (iD *ab
}

\begin{abstract}
A heat-triggered drug release strategy based on ultrasound-assisted mild hyperthermia and thermosensitive liposomes has emerged as a promising option to enable spatiotemporally controlled, efficient drug delivery for cancer treatment. However, consequential thermal vascular damage may decrease drug extravasation into tumor tissue and affect the therapeutic efficacy of follow-up treatments. To overcome this limitation, we explored a non-thermal acoustic treatment. Doxorubicin (DOX), an anticancer drug, was encapsulated in fatty acid-conjugated, elastin-like peptide (FELP)-bearing thermosensitive liposomes (FTSLs) for comparison of two treatments and their therapeutic implications. DOX-FTSLs had an average hydrodynamic size of $134.9 \mathrm{~nm}$ with a unimodal distribution. Their thermosensitivity allowed the triggering of rapid DOX release at $42{ }^{\circ} \mathrm{C}$, a mild-hyperthermia relevant temperature, with sustained DOX release at $37^{\circ} \mathrm{C}$. Interestingly, non-thermal acoustic treatment right after systemic administration of DOX-FTSLs into tumor-bearing mice led to higher tissue penetration without permanent vascular damage, greater intratumoral DOX accumulation, and similar therapeutic efficacy to thermal treatment. Overall, non-thermal acoustic treatment may be a safe alternative to thermosensitive liposome-involved hyperthermia for liposomal chemotherapy.
\end{abstract}

\section{Introduction}

Nanoparticles have been extensively studied as a delivery system for anticancer drugs to address pharmaceutical challenges ${ }^{1}$ such as dose-limiting systemic toxicity, rapid clearance ${ }^{2,3}$ and poor targeting of the tumoral region., ${ }^{4,5}$ That is primarily because nanoparticles selectively accumulate at tumor sites in a size-dependent manner through highly-permeable endothelial gaps existed over defective angiogenic tumor vasculature. ${ }^{6,7}$ A deficient tumor lymphatic drainage system allows the particles to be also retained more in tumors than in normal tissue. These features support the concept of passive cancer targeting through the enhanced permeability and retention effect., ${ }^{\mathbf{8}, 9}$

of the nanoparticles, liposomes have attracted much attention as a versatile drug carrier because they are biocompatible, biodegradable, and amenable to incorporating biologically active drug molecules. ${ }^{10,11}$ However, the short blood circulation

\footnotetext{
${ }^{a}$ Department of Health Sciences and Technology, SAIHST, Sungkyunkwan University, Seoul 06351, Republic of Korea. E-mail: jhpark1@skku.edu; Fax: +82-31-299-6857; Tel: $+82-31-290-7288$

${ }^{b}$ School of Chemical Engineering, College of Engineering, Sungkyunkwan University, Suwon 16419, Republic of Korea

'Medical Device Research Center, Research Institute for Future Medicine, Samsung Medical Center, Seoul 06351, Republic of Korea

${ }^{d}$ Samsung Biomedical Research Institute, Samsung Advanced Institute of Technology, Samsung Electronics Co., Ltd., Seoul 06351, Republic of Korea

$\dagger$ These authors contributed equally to this work.
}

time and mechanism of sustained drug release through passive diffusion of liposomes may not bring cytotoxic local drug concentrations to tumors. ${ }^{11}$ Therefore, efforts have focused on developing stimuli-sensitive liposomes that can induce rapid, target-specific release of drugs in response to stimuli such as $\mathrm{pH},{ }^{12,13}$ temperature, ${ }^{14,15}$ ultrasound, ${ }^{16-18}$ and light. ${ }^{19-21}$ In particular, thermosensitive liposomes have been intensively investigated in the recent decades for heat-triggered drug release in conjunction with mild hyperthermia induced by ultrasound or radiofrequency. ${ }^{10,22,23}$ Mild hyperthermia causing local heating of tumor tissue to around $42{ }^{\circ} \mathrm{C}$ improves the efficiency of chemotherapy by temporarily increasing tumor blood flow and microconvection in the tumor interstitium. ${ }^{22,24}$ If thermosensitive liposomes are broken in mild hyperthermia conditions, a burst release of the drug can be induced selectively at the desired site. ${ }^{24,25}$ Recently, we demonstrated enhanced antitumor efficacy with a synergistic combination of mild hyperthermia and fatty acid-conjugated elastin-like polypeptide (FELP)-bearing thermosensitive liposomes (FTSLS) that have a long blood circulation time and selective collapse at or above $40{ }^{\circ} \mathrm{C} ., 26$ However, after hyperthermia treatment by high intensity focused ultrasound (HIFU), thermal vascular damage has been observed as reported elsewhere. ${ }^{27}$ We hypothesized that although mild hyperthermia temporarily improves the efficacy of liposomal chemotherapy, the resulting irreversible thermal damage can inhibit extravasation of the drug to the tumor site. This consequence may decrease the overall therapeutic efficacy of follow-up treatments. 


\section{Thermal Acoustic treatment (Mild hyperthermia)}

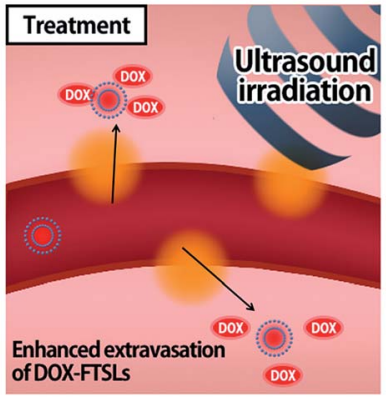
FTSLs.

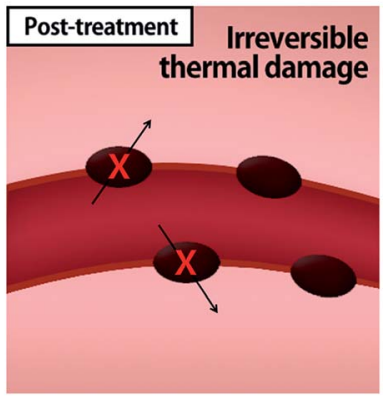

\section{Non-thermal Acoustic treatment}

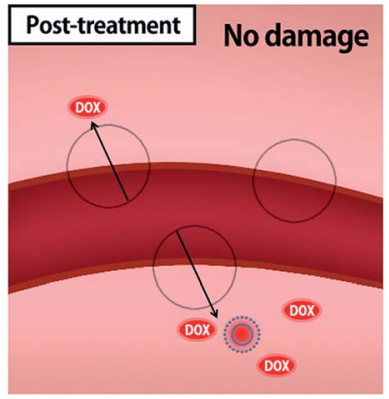

Fig. 1 Schematic illustration representing the drug delivery mechanisms of thermal and non-thermal acoustic treatment using DOX-

Herein, we devised non-thermal acoustic treatments by adjusting HIFU power, and compared its treatment effects on the in vivo behavior of doxorubicin (DOX)-loaded FTSLs (DOX-FTSLs) with thermosensitive liposome-involved hyperthermia. Thermal and non-thermal acoustic treatments were harnessed to improve the therapeutic efficacy of liposomal chemotherapy via different drug delivery mechanisms (Fig. 1). With ultrasound-induced hyperthermia, liposome collapse near tumor tissue triggers a burst release of drugs and irreversible damage occurs in the tumor tissue microvasculature. ${ }^{\mathbf{4 , 2}}$ Unlike thermal ultrasound, nonthermal acoustic treatment temporarily increases the permeability of tumor vessels via an acoustic cavitation effect ${ }^{29}$ without a rise in temperature and liposome collapse so that undisrupted liposomes are expected to be efficiently extravasated into tumor tissue. To the best of our knowledge, this is the first report to demonstrate superior antitumor drug delivery potential and comparable therapeutic efficacy of the non-thermal acoustic treatment compared with the hyperthermia in vivo.

\section{Experimental}

Materials

1,2-Dipalmitoyl-sn-glycero-3-phosphocholine (DPPC), 1,2-distearoyl-sn-glycero-3-phospho-ethanolamine- $N$-[methoxy(polyethylene glycol)-2000] (DSPE-PEG), 1,2-dipalmitoyl-sn-glycero-3phosphoethanolamine (DPPE) and cholesterol were obtained from Avanti Polar Lipid, Inc. (Alabaster, USA). FELP $\left(\mathrm{C}_{18} \mathrm{H}_{37^{-}}\right.$ $[\text { VPGVG }]_{3}-\mathrm{NH}_{2}$ ) was purchased from American Peptide, Inc. (Sunnyvale, USA). The near-infrared fluorescent (NIRF) dye,
Cy5.5 was obtained from Amersham Biosciences (Piscataway, USA). DOX was purchased from Sigma-Aldrich (St. Louis, USA). All other chemicals were analytical grade. The EMT6 cell line was purchased from the American Type Culture Collection (Rockville, USA). For cell culture, Dulbecco's modified Eagle medium (DMEM), Dulbecco's phosphate-buffered saline (PBS), trypsin-EDTA, and fetal bovine serum (FBS) were purchased from Invitrogen (Waltham, USA). All experiments involving live animals were performed in compliance with the relevant laws and institutional guidelines of Korea Institute of Sciences and Technology, and institutional committees approved all experimental protocols (KIST-2016-006).

\section{Preparation of DOX-FTSLs}

DOX-FTSLs were produced by a thin film hydration method as described previously. ${ }^{\mathbf{4} 26}$ FTSLs were formulated with DPPC : DSPEPEG : cholesterol : FELP at a $55: 2: 15: 0.4125$ molar ratio. Lipids, cholesterol, and FELP were dissolved in chloroform, and solvent was evaporated to prepare a thin film, which was hydrated with $250 \mathrm{mM}$ ammonium sulfate solution ( $\mathrm{pH} 4.0)$ by rigorous vortexing and sonication. The resulting solution was extruded through a $100 \mathrm{~nm}$ pore polycarbonate membrane to facilitate formation of liposomes with homogeneous size distribution. The ammonium sulfate buffer was replaced with $25 \mathrm{mM}$ Tris $\cdot \mathrm{HCl}(\mathrm{pH}$ 9.0) using a pre-packed Sephadex (G-25M) column. DOX was then loaded into the liposomes by adding it to liposome suspensions and incubating at $37^{\circ} \mathrm{C}$ for $1 \mathrm{~h}$. To remove free DOX and adjust $\mathrm{pH}$ to neutral, a pre-packed Sephadex (G-25M) column was used with PBS (pH 7.4) as eluent. Cy5.5-labeled DOX-FTSLs (Cy5.5-DOXFTSLs) were also prepared by using Cy5.5-conjugated DPPE in the FTSLs formulation for in vivo NIRF imaging.

\section{Characterization of DOX-FTSLS}

The hydrodynamic size and size distribution of DOX-FTSLs were measured by dynamic light scattering (Zetasizer Nano ZS90; Malvern Instruments, Worcestershire, UK) with a He-Ne laser operated at $633 \mathrm{~nm}$ and $25^{\circ} \mathrm{C}$ in the $173^{\circ}$ backscatter mode. Size measurements were triplicate. The concentration of DOX was determined by its absorbance at $480 \mathrm{~nm}$ using a UV-vis spectrometer (G1103A; Agilent, Worcestershire, USA) after dissolving in a water/tetrahydrofuran mixture (v/v). The DOX content and DOX loading efficiency of DOX-FTSLs were calculated by the following formulas:

$$
\text { Loading content }(\%)=\frac{\text { amount of loaded drug }}{\text { amount of liposome }} \times 100 \%
$$

$$
\text { Loading efficiency }(\%)=\frac{\text { amount of loaded drug }}{\text { amount of drug in feed }} \times 100 \%
$$

\section{In vitro DOX release test of DOX-FTSLS}

The temperature-dependent drug release profiles of DOX-FTSLS were studied by measuring DOX absorbance with a UV-vis spectrometer (G1103A; Agilent, Worcestershire, USA). $0.5 \mathrm{~mL}$ 
of DOX-FTSLs solution ( $1 \mathrm{mg} \mathrm{mL} \mathrm{mL}^{-1}$ ) was loaded into a dialysis membrane bag (MWCO = 3.5 kDa), immersed in $10 \mathrm{~mL}$ of $10 \%$ serum-PBS buffer ( $\mathrm{pH} 7.4)$ and gently shaken in a water bath $\left(37{ }^{\circ} \mathrm{C}\right.$ and $\left.42{ }^{\circ} \mathrm{C}, 100 \mathrm{rpm}\right)$. At 5, 15, 30, 60, and $120 \mathrm{~min}$, the amount of released DOX in medium was determined by measuring the absorbance at $480 \mathrm{~nm}$. The separate sample groups were prepared for each pre-set time points.

\section{Experimental settings for acoustic treatments}

A small animal HIFU system (VIFU 2000, Alpinion, Seoul, Korea) was used for ultrasound treatments to tumor tissues (Fig. 2). Mice were anesthetized via intraperitoneal injection of avertin (2,2,2-tribromoethanol) and fixed on a custom-made holder for treatment. The transducer and target tumor were aligned in a water bath filled with degassed water and maintained at $37^{\circ} \mathrm{C}$. HIFU parameters were optimized by iterative adjustment based on direct in vivo temperature measurements using a thermocouple wire $(50 \mu \mathrm{m}$ in diameter, Physitemp Instrument Inc., Clifton, USA) inserted into tumor tissues. For thermal treatment, positions with seven or eight HIFU foci were preset on tumors at $2.5 \mathrm{~mm}$ intervals; each was sequentially exposed to HIFU for $300 \mathrm{~s}$ (power: $20 \mathrm{~W}$, duty cycle: $15 \%$, pulse repetition frequency: $15 \mathrm{~Hz}$ ) to maintain tumor tissues at $42{ }^{\circ} \mathrm{C}$. For nonthermal acoustic treatment, power was adjusted to $10 \mathrm{~W}$ to maintain tumor tissue at $37^{\circ} \mathrm{C}$ while keeping other parameters the same as for thermal treatment. In an animal study $(n=3)$, DOX-FTSLs ( $5 \mathrm{mg}$ DOX per $\mathrm{kg}$ ) were systemically administered through the tail vein of mice, and HIFU (thermal or nonthermal acoustic treatment) was immediately used to irradiate tumors. To evaluate the therapeutic efficacy of DOX-FTSLs with thermal or non-thermal acoustic treatment, tumor areas were irradiated again under thermal acoustic treatment conditions at 12 hours post injection for burst release of DOX by disruption of FTSLs already deposited in tumor tissues.

\section{Tumor implantation}

For animal studies, an allograft tumor model was established using EMT6 mouse mammary tumor cells with five-week-old male BALB/c nude mice. For cell cultivation, DMEM with 15\% (v/v) FBS, $100 \mu \mathrm{g} \mathrm{mL} \mathrm{m}^{-1}$ penicillin, and $100 \mu \mathrm{g} \mathrm{mL} \mathrm{m}^{-1}$ streptomycin was used. Tumor models were prepared by subcutaneously injecting $200 \mu \mathrm{L}$ of cell suspension $\left(1 \times 10^{6}\right.$ EMT6 cells for each mouse) into the left thigh. Tumors were grown for 10 days to $150-200 \mathrm{~mm}^{3}$.

\section{Ex vivo histological analysis of tumor tissues}

For histological staining, tumors were collected from mice groups 1 hour post acoustic treatment. Tissues were fixed with $4 \%$ paraformaldehyde and embedded into paraffin. Paraffinembedded tumor tissues were sectioned in $4 \mu \mathrm{m}$ slices, stained with hematoxylin and eosin (H\&E), and observed by bright-field microscopy (BX 51; Olympus, Melville, USA).

\section{In vivo biodistribution of DOX-FTSLs}

In vivo biodistribution of DOX-FTSLs was evaluated for each treatment protocol using an explore Optix system (ART Advanced Research Technologies Inc., Montreal, Canada) with laser set to output power $10 \mu \mathrm{W}$ for $0.3 \mathrm{~s}$ per point, after intravenous injection of Cy5.5-DOX-FTSLs ( $5 \mathrm{mg}$ DOX per $\mathrm{kg}$ ) into tumor-bearing mice. At predetermined time points, fluorescence images of mice were obtained with excitation at $635 \mathrm{~nm}$ and detection at $693 \mathrm{~nm}$ for Cy5.5. Quantification of NIRF images was done by OptiView software (ART Advanced Research Technologies Inc., Montreal, Canada).

\section{In vivo fluorescence images of tumor vessels}

To qualitatively and quantitatively assess extravasation of DOX and Cy5.5-DOX-FTSLs into tumors in mice models, NIRF images of tumor vessels were observed by an optical live imaging system (OV-100; Olympus Corp., Tokyo, Japan). Cy5.5DOX-FTSLs ( $5 \mathrm{mg}$ DOX per $\mathrm{kg}$ ) were systemically injected into tail vein of tumor-bearing mice with immediate thermal or nonthermal acoustic treatment on tumor tissues. At $12 \mathrm{~h}$ post injection, tumoral skin of EMT-6-bearing mice was removed for fluorescent detection. Accumulation of Cy5.5-DOX-FTSLs and DOX around tumor vessels was visualized and quantified using an RFP filter (excitation $=535-555 \mathrm{~nm}$; emission $=570-623$ $\mathrm{nm}$ ) and Cy5.5 filter (excitation $=620-650 \mathrm{~nm}$ and emission $=$ (a)

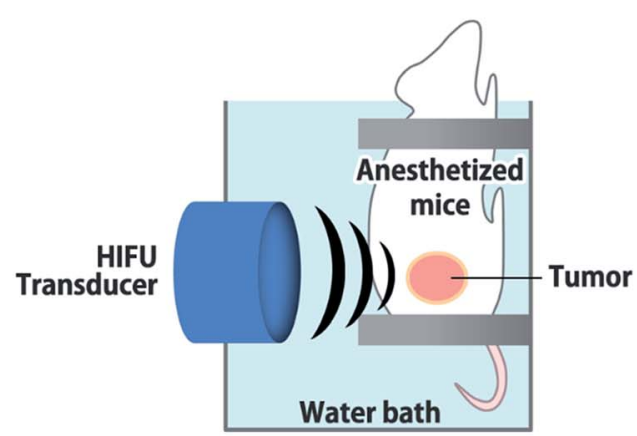

(b)

\begin{tabular}{|c|c|}
\hline $\begin{array}{c}\text { Thermal } \\
\text { acoustic treatment }\end{array}$ & $\begin{array}{c}\text { Non-thermal } \\
\text { acoustic treatment }\end{array}$ \\
\hline Dox burst release & $37^{\circ} \mathrm{C}$ \\
\hline
\end{tabular}

Fig. 2 Schematic representation of the experimental set up for acoustic treatments. (a) Alignment of HIFU transducer with murine tumor mass. (b) Experimental conditions for thermal or non-thermal acoustic treatment. 
680-710 nm). NIRF intensities were quantitatively determined using Image-Pro Plus software 4.0 (Media Cybernetics, Rockville, USA).

\section{Antitumor efficacy of DOX-FTSLs}

Mice were divided into five groups: saline, free DOX, DOXFTSLs, DOX-FTSLs (thermal), DOX-FTSLs (non-thermal). Tumor-bearing mice for control groups (saline, free DOX, and DOX-FTSLs) were not exposed to HIFU. DOX-FSTLs (thermal) group and DOX-FSTLs (non-thermal) group were irradiated by HIFU immediately after systemic injection of DOX-FSTLs $(5 \mathrm{mg}$ DOX per kg). After 12 hours, mice underwent thermal acoustic treatment to burst liposomes accumulated in tumors. Upon drug administration followed by HIFU treatment, tumor growth was recorded over 12 days. Tumor size was measured by Vernier

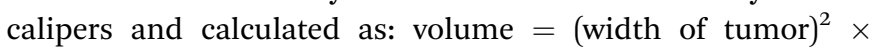
(length of tumor) $\times \pi / 6$. Values were presented as mean with standard errors for groups of $n=3$.

\section{Statistical analysis}

One way analysis of variance (ANOVA) was conducted to determine statistical significance of differences between groups, and marked with an asterisk (*) in figures if $p$ value was less than 0.05 .

\section{Results and discussion}

\section{Preparation and characterization of DOX-FTSLS}

To compare the therapeutic potency of thermal acoustic treatment with a non-thermal alternative, we prepared DOX-FTSLs, which can be ruptured under thermal conditions. FTSLs were composed of two phospholipids (DPPC and DSPE-PEG) ${ }^{30}$ with cholesterol as the steric stabilizer of the bilayer ${ }^{31}$ and FELP as the transition temperature adjuster. ${ }^{32}$ DOX, a model chemotherapeutic drug, was encapsulated in FTSLs to produce DOXFTSLs. After purification, DOX content of DOX-FTSLs was
9.3\% and DOX loading efficiency was 93.2\%. DOX-FTSLs had a mean diameter of $134.97 \mathrm{~nm}$ and polydispersity index value of 0.417 with a unimodal size distribution (Fig. 3a), consistent with our previous report. ${ }^{4}$

\section{In vitro temperature-dependent release profile of DOX-FTSLs}

DOX release profiles of DOX-FTSLs were obtained as a function of time at two temperatures mimicking body temperature (37 ${ }^{\circ} \mathrm{C}$ ) and mild hyperthermia $\left(42^{\circ} \mathrm{C}\right)$ (Fig. 2b). At $37{ }^{\circ} \mathrm{C}$, DOXFTSLs released about $44 \%$ of encapsulated DOX within $2 \mathrm{~h}$, exhibiting first-order release kinetics. The DOX release profiles of DOX-FTSLs were obtained as a function of time at two different temperatures mimicking body temperature $\left(37^{\circ} \mathrm{C}\right)$ and mild hyperthermia $\left(42^{\circ} \mathrm{C}\right)$ (Fig. 3b). At $37{ }^{\circ} \mathrm{C}$, DOX-FTSLs released about $44 \%$ of the encapsulated DOX within $2 \mathrm{~h}$, exhibiting first-order release kinetics. Conversely, at $42{ }^{\circ} \mathrm{C}$, a substantial amount of DOX (82.75\%) released from the DOXFTSLs was observed within the initial $5 \mathrm{~min}$, and the amount of DOX released was soon saturated to $96 \%$ of the total encapsulated DOX amount. The thermosensitive burst release under hyperthermia conditions might have been caused by the destabilization of the liposomal membrane with conformational changes of FELPs at $42{ }^{\circ} \mathrm{C}$, which is higher than the transition temperature of the DOX-FTSLs $\left(40{ }^{\circ} \mathrm{C}\right) .{ }^{13}$ The DOXFTSLs maintained liposomal structure better at the nonthermal physiological temperature of $37{ }^{\circ} \mathrm{C}$ compared with the thermal temperature of $42{ }^{\circ} \mathrm{C}$. Based on these results, in vivo ultrasound-assisted hyperthermia was also expected to trigger the exclusive release of DOX with collapse of DOX-FTSLs in the focal region, with non-thermal acoustic treatment reducing DOX leakage.

\section{Evaluation of tumor tissue damage after acoustic treatments}

To assess damage to tumor tissues after thermal or non-thermal acoustic treatment, we tuned acoustic parameters to maintain tumor tissues around $42{ }^{\circ} \mathrm{C}$ for thermal and $37{ }^{\circ} \mathrm{C}$ for non- (a)

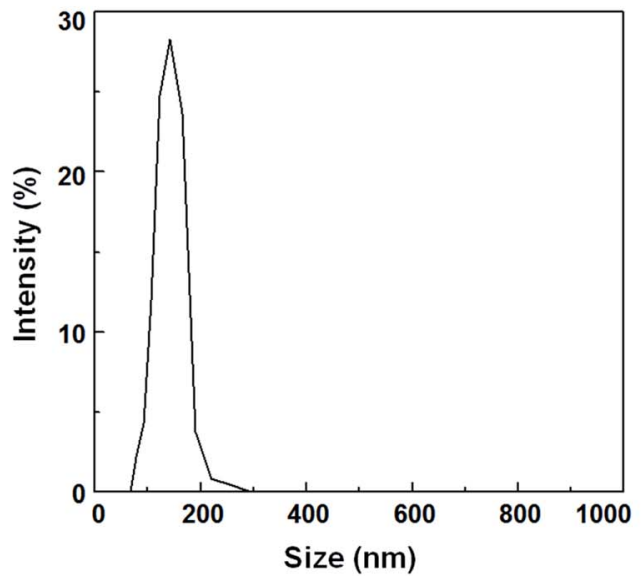

(b)

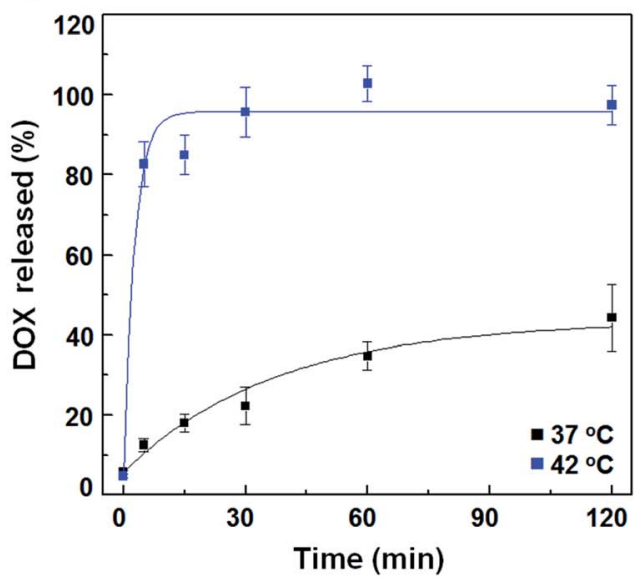

Fig. 3 Characterization of DOX-FTSLs. (a) Size distribution of DOX-FTSLs in PBS (pH 7.4). (b) In vitro DOX release profiles of DOX-FTSLs at $37^{\circ} \mathrm{C}$ and at $42{ }^{\circ} \mathrm{C}$. Error bars represent standard deviation $(n=3)$. 
thermal. During ultrasound irradiation, we monitored tumor tissue temperatures to confirm proper control. Recorded average temperatures for thermal treatment were $42.5 \pm$ $0.36^{\circ} \mathrm{C}$, sufficient to disrupt DOX-FTSLs. Average temperatures for non-thermal treatment were $37.7 \pm 0.4{ }^{\circ} \mathrm{C}$. We considered fluctuations in temperatures acceptable for experiments since differences from target temperatures were less than $1{ }^{\circ} \mathrm{C}$, which still met the purpose of the specific temperatures. In histological analysis, only H\&E-stained mouse tumor tissues from ultrasound focal regions treated under thermal conditions were swollen from edema, implying substantial damage (Fig. 4). No significant damage was observed in the control groups including the non-thermal treatment group.

\section{In vivo biodistribution}

To compare the effects of acoustic treatment methods on tumor-targeting efficiency of DOX-FTSLs, in vivo biodistributions were investigated (Fig. 5). DOX-FTSLs were tracked using a NIRF imaging system via labeling with Cy5.5, systemic administration into EMT6 tumor-bearing mice, and

\section{No treatment}
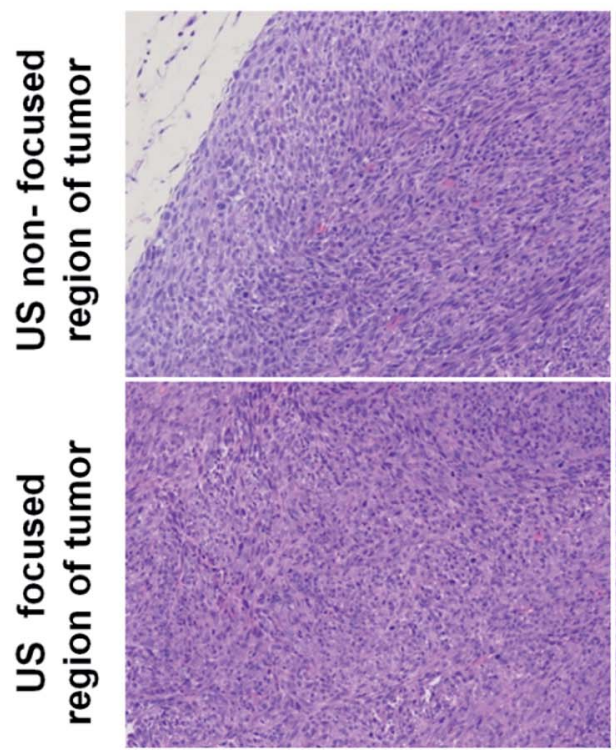

\section{Thermal} treatment
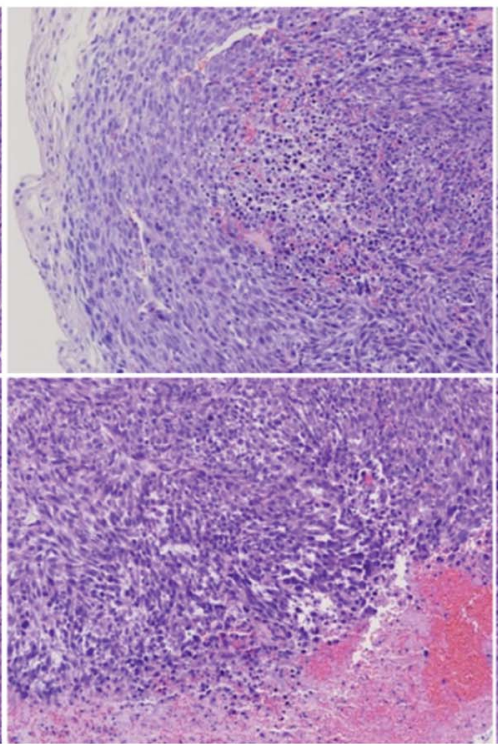

\section{Non-thermal treatment}

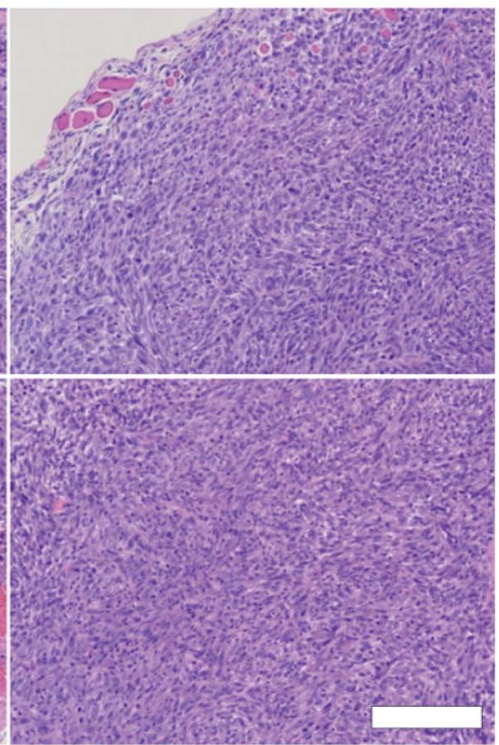

Fig. 4 H\&E staining images of tumor tissues excised from treatment groups. Scale bar represents $200 \mu \mathrm{m}$.

(a)

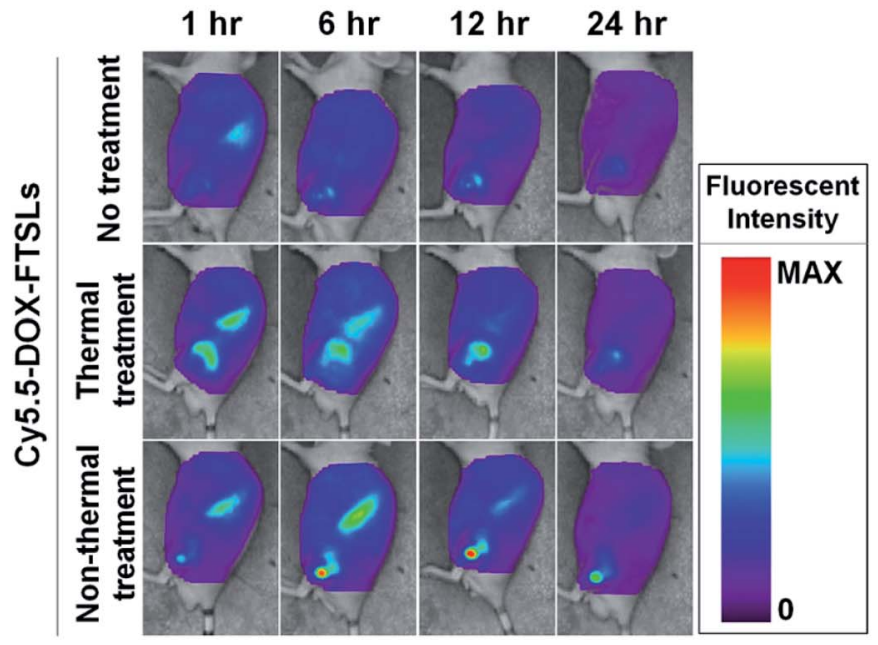

(b)

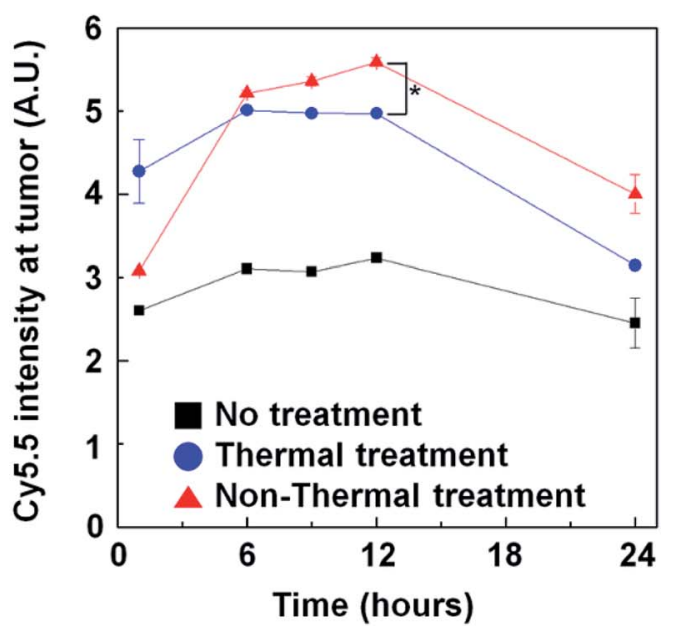

Fig. 5 (a) In vivo biodistribution of Cy5.5-labeled DOX-FTSLs by treatment group. (b) Normalized fluorescent intensities at tumor tissues as a function of time. Error bars represent standard error $\left(n=3 ;{ }^{*} p<0.05\right.$ calculated by one-way ANOVA). 
monitoring for $24 \mathrm{~h}$. Overall, thermal and non-thermal acoustic treatment improved intratumoral accumulation of Cy5.5-DOXFTSLs. After the first $1 \mathrm{~h}$, fluorescent signal intensity at tumor site of the mice received the thermal or non-thermal treatment was 1.64-fold or 1.18-fold higher, respectively, than that of the no treatment group. No groups showed drastic changes in signal intensity at tumor sites for the time period between $6 \mathrm{~h}$ and $12 \mathrm{~h}$ post injection. In the non-thermal group, tumor signal intensity increased to 1.75-fold higher than the no-treatment group, and was higher than the thermal group at 6 hours after Cy5.5-DOX-FTSL injection. The non-thermal group signal gradually increased until 12 hours post injection, showing significantly greater signal intensity than the thermal group. These results indicated that although thermal treatment resulted in higher intratumoral accumulation of Cy5.5-DOX-FTSLs than non-thermal treatment at 1 hour post injection, after five hours, non-thermal treatment had higher accumulation. Observed thermal damage on tumor tissue including vasculature shown in Fig. 4 might correlate with less improvement with thermal treatment on a tumor-specific biodistribution compared to non-thermal treatment.

\section{Evaluation of vessel permeability change after acoustic} treatments

To compare the effect of thermal and non-thermal treatment on extravasation of the DOX-FTSLs and DOX, in vivo NIRF live imaging of tumor tissue was conducted at $12 \mathrm{~h}$ post injection of Cy5.5-DOX-FTSLs (Fig. 6a). We observed tumor blood vessels closely, and visualized DOX-FTSLs using Cy5.5 and DOX using its own fluorescence. By bright field image of the thermal treatment group, tumor vessels showed damage with risk of reducing or blocking tumor blood flow because of thrombosis. This effect would decrease the supply of drugs into tumors. A negligible amount of fluorescent signal corresponding to extravasated DOX-FTSLs was seen near damaged vessels while the fluorescent signal of released DOX was widespread. This is probably because the substantial amount of DOX is first released from the DOX-FTSLs upon liposome rupture near tumor tissue and then extravasation occurs. Conversely, no vessel damage was observed in tumor tissues receiving nonthermal acoustic treatment and the DOX and DOX-FTSL fluorescent signal was entirely dispersed and overlapping. These

(a)

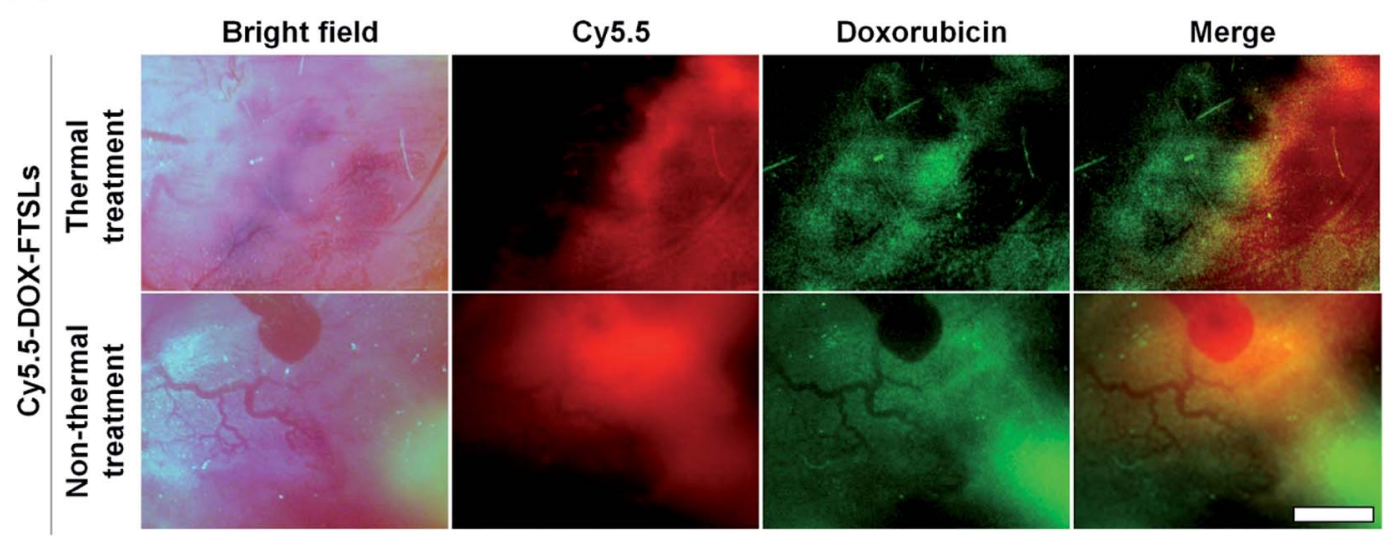

(b)

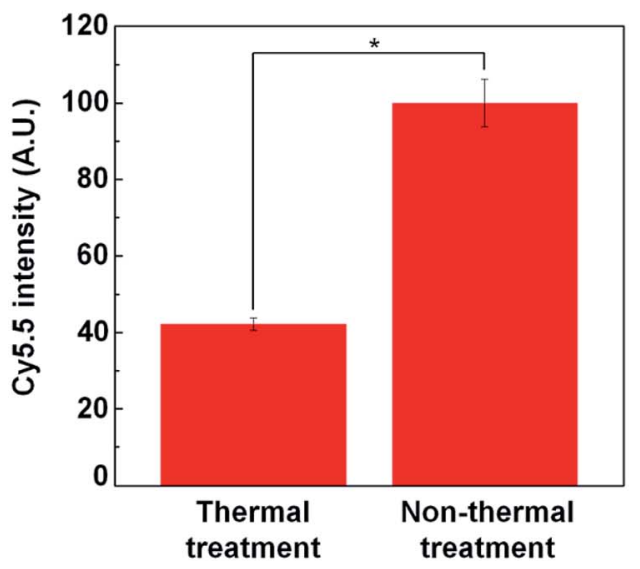

(c)

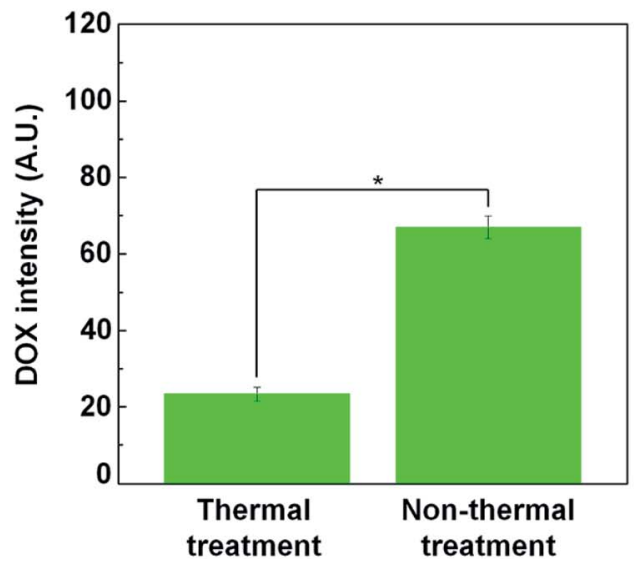

Fig. 6 (a) In vivo NIRF images of tumor vessels and tissues at $12 \mathrm{~h}$ after intravenous injection of Cy5.5-labeled DOX-FTSLs combined with thermal or non-thermal acoustic treatment (red: Cy5.5, green: DOX). Scale bar represents $150 \mu \mathrm{m}$. (b) Quantification of liposome accumulation at tumor tissues with Cy5.5 NIRF images. (c) Quantification of DOX accumulation at tumor tissues with DOX NIRF images. Error bars represent standard deviation ( $n=3$; * $p<0.05$ calculated by one-way ANOVA). 
results suggested that tumor vessel damage from thermal treatment hindered accumulation of DOX-FTSLs while nonthermal treatment accommodated accumulation of both DOX and DOX-FTSLs without producing recognizable vessel damage. Quantitative analysis of intratumoral accumulation showed that, under nonthermal acoustic treatment conditions, 2.87-fold more DOX and 2.37-fold more DOX-FTSLs extravasated into tumoral regions compared with thermal conditions (Fig. 5b). These results verified our main hypothesis that thermal vascular damage under hyperthermia conditions inhibits extravasation of drug to tumor sites. Based on our experiments, non-thermal treatment was safer and a better tool for delivering drugs to tumoral regions.

\section{In vivo antitumor efficacy}

Although non-thermal treatment has been favored for tumortargeted drug delivery, whether it improves overall therapeutic efficacy compared with control groups still needed to be assessed. To evaluate the therapeutic efficacy of the treatment methods, in vivo tumor growth was examined by measuring tumor volumes over 12 days (Fig. 7). After administration of DOX-FTSLs, free DOX or saline at a dose of $5 \mathrm{mg}$ DOX per $\mathrm{kg}$, thermal or non-thermal acoustic treatment was immediately carried out as indicated. Quantified intratumoral DOX could be either encapsulated in the FTSLs or released from them. To reflect the drug action of total accumulated DOX, we needed to induce burst release of encapsulated DOX. Therefore, a second thermal acoustic treatment was applied to tumor tissues of acoustically-treated samples at $12 \mathrm{~h}$ post injection, when intratumoral DOX-FTSL accumulation was maximal and DOXFTSLs were systemically circulating in the bloodstream (Fig. 5). Non-thermal acoustic treatment resulted in tumor growth inhibition that was comparable to thermal treatment with the lowest average final tumor volume; it was only $37.7 \%$ of the volume with DOX-FTSLs in the no acoustic treatment group and $62.7 \%$ of the volume with DOX-FTSLs in the thermal

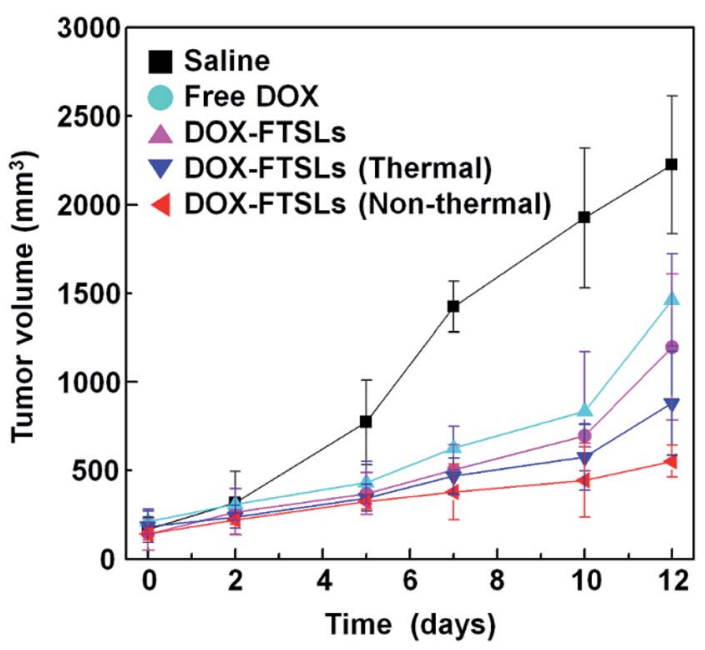

Fig. 7 Antitumor efficacy of DOX-FTSLs by treatment group. Error bars represent standard deviation $(n=3)$. treatment group at the same dose. Thus, non-thermal acoustic treatment, which resulted in effective drug accumulation without irreversible vessel damage, may be a safe alternative to thermosensitive liposome-involved hyperthermia.

\section{Conclusion}

Hyperthermia-assisted liposomal chemotherapy is considered a synergistic combinational approach, since thermally triggered, tumor site-specific drug release is ideal for improving overall therapeutic efficacy while minimizing possible side effects of chemotherapeutic drugs. However, vascular damage often observed in HIFU focal regions of treated tumor tissues might be a limitation to necessary follow-up treatments that are common for conventional chemotherapy. We visualized tumor vessel damage caused by thermal acoustic treatment, and quantitatively assessed the resultant decrease in intratumoral accumulation of DOX-FTSLS and DOX compared with non-thermal treatment. Our results demonstrated the superior drug delivery potential and comparable therapeutic efficacy of non-thermal acoustic treatment compared with hyperthermia, suggesting the former as a safe alternative to partially replace hyperthermia in an overall treatment regimen. Though much work needs to be done to provide detailed guidelines for clinical applications, this simple strategy had the potential to improve clinical practice by offering more cancer treatment options.

\section{Acknowledgements}

This work was financially supported by the Basic Science Research Programs (NRF-2016R1A6A3A11932550 and NRF-2010-0027955), and the Global Research Laboratory Program (NRF2016K1A1A2A02942563) through the National Research Foundation (NRF) of Korea. Additionally, it was supported by the Program for Returners into R\&D (WISET-2016-253) through the NRF and the Center for Women in Science, Engineering and Technology.

\section{References}

1 E. Blanco, H. Shen and M. Ferrari, Nat. Biotechnol., 2015, 33, 941-951.

2 J. Harris and B. Robert, Nat. Rev. Drug Discovery, 2003, 2, 214221.

3 S. Jeon, H. Ko, R. N. Vijayakameswara, H. Y. Yoon, D. G. You, H. S. Han, W. Um, G. Saravanakumar and J. H. Park, RSC Adv., 2015, 5, 70352-70360.

4 J. M. Cha, D. G. You, E. J. Choi, S. J. Park, W. Um, J. Jeon, K. Kim, I. C. Kwon, J. C. Park, H. R. Kim and J. H. Park, J. Biomed. Nanotechnol., 2016, 12, 1724-1733.

5 M. Swierczewska, H. S. Han, K. Kim, J. H. Park and S. Lee, Adv. Drug Delivery Rev., 2016, 99, 70-84.

6 Y. Matsumoto, J. W. Nichols, K. Toh, T. Nomoto, H. Cabral, Y. Miura, R. J. Christie, N. Yamada, T. Ogura, M. R. Kano, Y. Matsumura, N. Nishiyama, T. Yamasoba, Y. H. Bae and K. Kataoka, Nat. Nanotechnol., 2016, 11, 533-538.

7 H. Cabral, Y. Matsumoto, K. Mizuno, Q. Chen, M. Murakami, M. Kimura, Y. Terada, M. R. Kano, K. Miyazono, M. Uesaka, 
N. Nishiyama and K. Kataoka, Nat. Nanotechnol., 2011, 6, 815-823.

8 H. Maeda, J. Wu, T. Sawa, Y. Matsumura and K. Hori, J. Controlled Release, 2000, 65, 271-284.

9 H. Maeda, H. Nakamura and J. Fang, Adv. Drug Delivery Rev., 2013, 65, 71-79.

10 V. P. Torchilin, Nat. Rev. Drug Discovery, 2005, 4, 145-160.

11 A. Puri, K. Loomis, B. Smith, J.-H. Lee, A. Yavlovich, E. Heldman and R. Blumenthal, Crit. Rev. Ther. Drug Carrier Syst., 2009, 26, 523-580.

12 E. Yuba, Y. Kanda, Y. Yoshizaki, R. Teranishi, A. Harada, K. Sugimura, T. Izawa, J. Yamate, N. Sakaguchi, K. Koiwai and K. Kono, Biomaterials, 2015, 67, 214-224.

13 Y. Zhao, W. Ren, T. Zhong, S. Zhang, D. Huang, Y. Guo, X. Yao, C. Wang, W. Q. Zhang, X. Zhang and Q. Zhang, J. Controlled Release, 2016, 222, 56-66.

14 L. Li, T. Hagen, M. Hossann, R. Süss, G. Rhoon, A. Eggermont, D. Haemmerich and G. Koning, J. Controlled Release, 2013, 168, 142-150.

15 K. Kono, M. Takashima, E. Yuba, A. Harada, Y. Hiramatsu, H. Kitagawa, T. Otani, K. Maruyama and S. Aoshima, J. Controlled Release, 2015, 216, 69-77.

16 S. Dromi, V. Frenkel, A. Luk, B. Traughber, M. Angstadt, M. Bur, J. Poff, J. Xie, S. K. Libutti, K. C. P. Li and B. J. Wood, Clin. Cancer Res., 2007, 13, 2722-2727.

17 J. VanOsdol, K. Ektate, S. Ramasamy, D. Maples, W. Collins, J. Malayer and A. Ranjan, J. Controlled Release, 2017, 247, 5563.

18 S. Rizzitelli, P. Giustetto, J. Cutrin, D. Castelli, C. Boffa, M. Ruzza, V. Menchise, F. Molinari, S. Aime and E. Terreno, J. Controlled Release, 2015, 202, 21-30.
19 T. Lajunen, L. Viitala, L. S. Kontturi, T. Laaksonen, H. Liang, E. Vuorimaa-Laukkanen, T. Viitala, X. Guével and M. Tliperttula, J. Controlled Release, 2015, 203, 85-98.

20 K. Carter, S. Shao, M. Hoopes, D. Luo, B. Ahsan, V. Grigoryants, W. Song, H. Huang, G. Zhang, R. Pandey, J. Geng, B. Pfeifer, C. Scholes, J. Ortega, M. Karttunen and J. Lovell, Nat. Commun., 2014, 3546.

21 G. Wu, A. Mikhailovsky, H. A. Khant, C. Fu, W. Chiu and J. A. Zasadzinski, J. Am. Chem. Soc., 2008, 130, 8175-8177.

22 G. Koning, A. Eggermont, L. Lindner and T. Hagen, Pharm. Res., 2010, 27, 1750-1754.

23 S. Mura, J. Nicolas and P. Couvreur, Nat. Mater., 2013, 12, 991-1003.

24 Z. S. Al-Ahmady, W. T. Al-Jamal, J. V. Bossche, T. T. Bui, A. F. Drake, A. J. Mason and K. Kostarelos, ACS Nano, 2012, 6, 9335-9346.

25 H. R. Kim, D. G. You, S.-J. Park, K.-S. Choi, W. Um, J.-H. Kim, J. H. Park and Y. S. Kim, Mol. Pharm., 2016, 13, 1528-1539.

26 S. M. Park, M. S. Kim, S.-J. Park, E. S. Park, K.-S. Choi, Y. S. Kim and H. R. Kim, J. Controlled Release, 2013, 170, 373-379.

27 F. Wua, W.-Z. Chena, J. Baia, J.-Z. Zoua, Z.-L. Wanga, H. Zhua and Z.-B. Wanga, Ultrasound Med. Biol., 2002, 28, 535-542.

28 A. Schroeder, R. Honen, K. Turjeman, A. Gabizon, J. Kost and Y. Barenholz, J. Controlled Release, 2009, 137, 63-68.

29 V. Frenkel, Adv. Drug Delivery Rev., 2008, 60, 1193-1208.

30 H. Lee, H. R. Kim and J. C. Park, Phys. Chem. Chem. Phys., 2014, 16, 3763-3770.

31 W. W. Sułkowski, D. Pentak, K. Nowak and A. Sułkowska, J. Mol. Struct., 2005, 744-747, 737-747.

32 S. M. Park, J. M. Cha, J. Nam, M. S. Kim, S. J. Park, E. S. Park, H. Lee and H. R. Kim, PLoS One, 2014, 9, e103116. 